Propriedades de invariância na observabilidade e controlabilidade de sistemas lineares a tempo contínuo com saltos markovianos

Alfredo Rafael Roa Narváez 
SERVIÇO DE PÓS-GRADUAÇÃO DO ICMC-USP

Data de Depósito: 02/03/2010

Assinatura:

\title{
Propriedades de invariância na observabilidade e controlabilidade de sistemas lineares a tempo contínuo com saltos markovianos
}

\author{
Alfredo Rafael Roa Narváez \\ Orientador: Prof. Dr. Eduardo Fontoura Costa
}

Dissertação apresentada ao Instituto de Ciências Matemáticas e de Computação - ICMC-USP, como parte dos requisitos para obtenção do título de Mestre em Ciências - Ciências de Computação e Matemática Computacional.

USP - São Carlos

Março/2010 


\section{Dedicatória}

Aos meus amados pais,

Yolima e Alfredo. 



\section{Agradecimentos}

Ao professor Eduardo Fontoura Costa pela excelente orientação oferecida na realização deste trabalho e pela motivação, colaboração e apoio nos momentos difíceis.

Aos meus pais Alfredo Roa Gómez e Yolima Narváez Camargo e às minhas irmãs Karen Roa Narváez e Yolima Roa Narváez por todo amor, suporte, carinho e incentivo.

A Yoli por ter dispendido amor, compreensão e paciência durante minha permanência aqui no Brasil.

Ao Conselho Nacional de Desenvolvimento Científico e Tecnológico - CNPq, pela bolsa concedida a este trabalho.

A todos os colegas, professores e funcionários do Instituto de Ciências Matemáticas e de Computação da Universidade de São Paulo. 



\section{Resumo}

ste trabalho estuda a observabilidade e controlabilidade para uma $\mathcal{E}$ classe de sistema dinâmico markoviano com saltos nos parâmetros, e uma coleção de matrizes de observabilidade e controlabilidade associadas. São explorados alguns resultados de invariância, bem como certas propriedades envolvendo essas matrizes. Uma dessas propriedades, relacionada com a coleção de matrizes de observabilidade é conhecida na literatura desta classe de sistemas, mas não há uma prova disponível. Esses resultados de invariância foram estendidos para o estudo da controlabilidade e sua respectiva coleção de matrizes associada, obtendo assim uma propriedade análoga ao caso da observabilidade. Os resultados obtidos são importantes para validar outros resultados existentes que se baseiam na propriedade referida. 



\section{Abstract}

his work studies observability and controlability of a class of Markov

systems with jumping parameters, and associated set of observability 2 and controlability matrices. We explore some invariance results regarding the state trajectory and certain properties involving those matrices. One of these properties, related with the collection of observability matrices, is employed in the literature of this class of systems, but there is no available proof. The invariance results are extended similarly to the context of controlability leading to a property that is analogous to the observability case. The obtained results are important to validate other existing results that rely on that property. 




\section{Sumário}

Resumo ..........................

Abstract . . . . . . . . . . . . . . v vii

Lista de Figuras . . . . . . . . . . . . . . . . . . . . . 3

1 Introdução $\quad 5$

1.1 Motivação . . . . . . . . . . . . . . . . . . . . . . . 5

1.2 Hipóteses . . . . . . . . . . . . . . . . . . . . . . . . . 8

1.3 Contribuições . . . . . . . . . . . . . . . . . . . . 99 9

1.4 Organização do Trabalho . . . . . . . . . . . . . . . . . . 11

$\begin{array}{llr}2 & \text { Preliminares } & 13\end{array}$

2.1 Notações . . . . . . . . . . . . . . . . . . . . . . . . . . . . . 13

2.2 Definições e resultados . . . . . . . . . . . . . . . . . . . . 15

3 Matriz de Observabilidade e propriedades de invariância $\quad 19$

3.1 Considerações e resultados iniciais . . . . . . . . . . . . . . . . 19

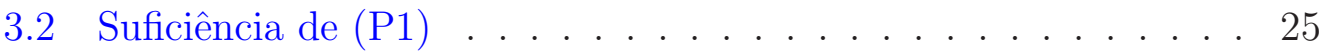

4 Extensões e/ou adaptações à matriz de Controlabilidade $\quad 29$

4.1 Adaptações e definições iniciais . . . . . . . . . . . . . . . . . . 29

4.2 Resultados auxiliares . . . . . . . . . . . . . . . . 30

4.3 Resultados de Controlabilidade. . . . . . . . . . . . . . . . . . 32

$\begin{array}{lll}5 & \text { Conclusões } & 37\end{array}$

Referências $\quad 42$ 


\section{Lista de Figuras}

1.1 Bastão em equilíbrio. . . . . . . . . . . . . . . . . . 7

3.1 Análise para a trajetória de $x(t)$ com respeito à distância a $\mathcal{N}\left(C_{j}\right) .18$

3.2 Duas diferentes perspectivas de 10 phase-plot para $x(t), 0 \leq t \leq S .24$ 


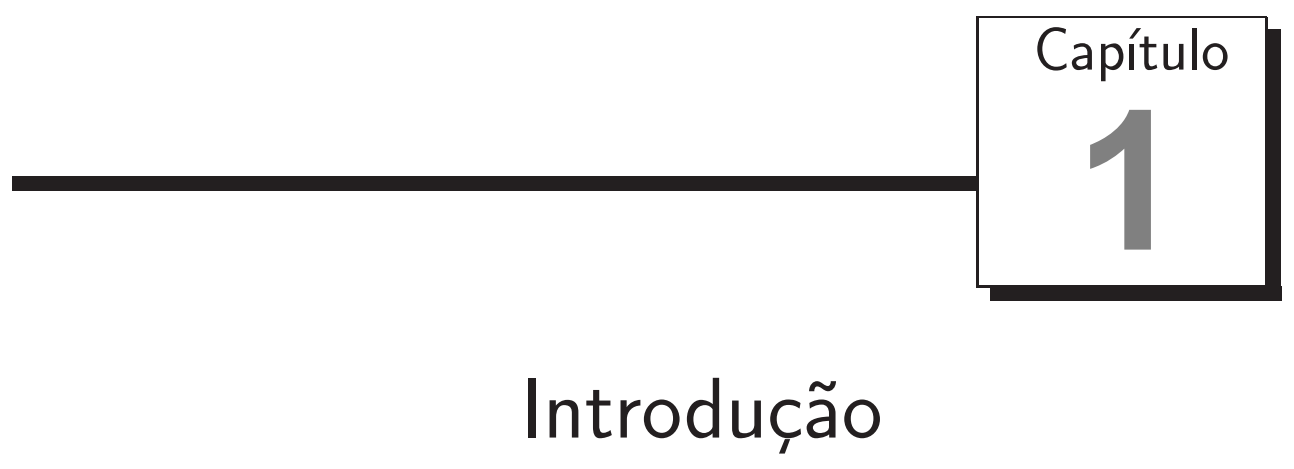

\subsection{Motivação}

Os conceitos duais de observabilidade e controlabilidade cumprem um papel importante na teoria de sistemas dinâmicos, caracterizando fundamentalmente as implicações dinâmicas da estrutura de entrada e saída deste tipo de sistemas. Tal estrutura pode influenciar significativamente os meios disponíveis para controle e filtragem. A noção de controlabilidade é relacionada com o fato que todos os estados podem ser alcançados. Nos Sistemas Lineares Determinísticos (SLD) temos uma importante caracterização da noção de controlabilidade. O sistema

$$
\dot{x}(t)=A x(t)+B u(t)
$$

é controlável se e somente se para um estado arbitrário $x\left(t_{1}\right)=x_{1}$ e qualquer estado arbitrário $x_{2}$ existe $t_{2}$ e uma entrada contínua por partes $u(t), t_{1} \leq t \leq$ $t_{2}$ tal que $x\left(t_{2}\right)=x_{2}$. O conceito de controlabilidade é equivalente a afirmar que a matriz de controlabilidade

$$
\mathbb{C}(A, B)=\left[B: A B: A^{2} B: \ldots \vdots A^{n-1} B\right]
$$

tem posto completo, onde $n$ é a dimensão de $x(t)$ e $A$ e $B$ são de dimensões apropriadas. Pode-se mostrar que, num sistema controlável, é possível direcionar o estado inicial a um estado específico qualquer em um número finito de 
passos, isto é num horizonte finito de tempo. Isto corresponde diretamente à noção intuitiva da capacidade de controlar os estados do sistema. Por outro lado, a observabilidade é importante na caracterização do comportamento do controle ótimo, por exemplo, garantindo a positividade do funcional de custo e a estabilidade do sistema controlado. Análogo à controlabilidade, temos que o sistema

$$
\begin{aligned}
& \dot{x}(t)=A x(t) \\
& y(t)=C x(t)
\end{aligned}
$$

é observável se e somente se existe $t_{1}>0$ tal que o conhecimento de $y(t)$, para todo $t, 0 \leq t \leq t_{1}$, é suficiente para determinar $x(0)$. O fato de ser o sistema observável é equivalente a afirmar que a matriz de observabilidade

$$
\mathbb{O}(A, C)=\left[C^{\prime}: A^{\prime} C^{\prime}: A^{\prime 2} C^{\prime}: \ldots \vdots A^{\prime n-1} C^{\prime}\right]^{\prime}
$$

tem posto completo, onde $A$ e $C$ são de dimensões apropriadas. Existe uma atenção muito grande para o estudo da controlabilidade e a observabilidade e há um bom número de resultados disponíveis na literatura, vide por exemplo Ji e Chizeck (1990); Bittanti et al. (1984); Ji e Chizeck (1988); Costa et al. (2006); Astolfi e Praly (2006); Davis e Vinter (1984); Gray e Mesko. (1999); Petersen (2002) só para mencionar uns poucos artigos em diferentes contextos.

Exemplo 1 (Bastão em equilíbrio). Um sistema sobre o qual sabemos, através da experiencia, que é controlável é o sistema do bastão em equilíbrio sobre uma base se movendo como ilustrado na Figura 1.1. Existem muitos problemas mecânicos tais como a manutenção de um satélite em órbita, o controle de um helicóptero e o controle de um foguete quando está sendo empurrado para cima entre outros, que têm um caráter semelhante. Vamos considerar o equilíbrio do bastão sobre a base como uma versão simples deste tipo de problemas. Verifiquemos então que esse sistema é controlável. Pode ser deduzido pelas leis de Newton que o sistema é governado pela equação (vide Luenberger (1979))

$$
\ddot{u}(t) \cos \theta(t)+L \ddot{\theta}(t)=g \sin \theta(t)
$$

onde g é a constante de gravitação. Também temos a relação

$$
x(t)=u(t)+L \sin \theta(t) .
$$

Assumindo que o bastão está próximo do repouso em uma posição vertical 
(com $\theta(t)$ muito pequeno), as duas equações (1.1) e (1.2) podem ser escritas em termos de $x(t)$ como

$$
\ddot{x}(t)=\frac{g}{L}[x(t)-u(t)] .
$$

Para facilitar a notação consideremos $L=1$. Então definindo a velocidade $v(t)=\dot{x}(t)$, o sistema tem a representação

$$
\left[\begin{array}{c}
\dot{x}(t) \\
\dot{v}(t)
\end{array}\right]=\left[\begin{array}{ll}
0 & 1 \\
g & 0
\end{array}\right]\left[\begin{array}{l}
x(t) \\
v(t)
\end{array}\right]+g\left[\begin{array}{c}
0 \\
-1
\end{array}\right] u(t) .
$$

A matriz de controlabilidade é

$$
\mathbb{C}(A, B)=-g\left[\begin{array}{ll}
0 & 1 \\
1 & 0
\end{array}\right]
$$

a qual claramente é de posto completo e portanto o sistema é controlável (sendo $g \neq 0)$.

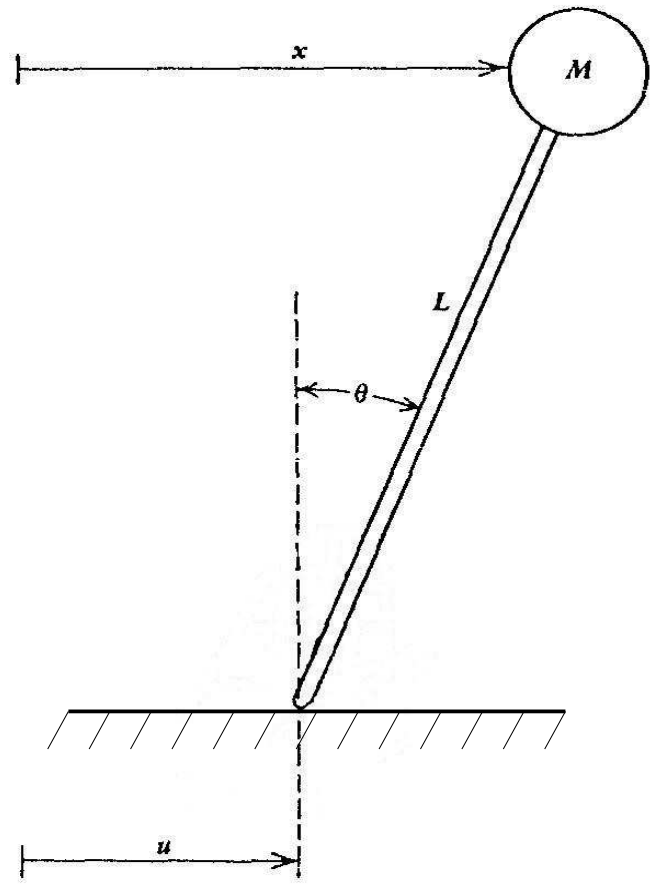

Figura 1.1: Bastão em equilíbrio.

Em particular, para Sistemas Lineares com Saltos de Markov (SLSM) (vide O. L. V. Costa e Marques (2000) para uma abordagem com teoria de opera- 
dores para SLSM e as referências alí incluídas, e do Val e Basar (1999); Saridis (1983); Siqueira e Terra (2004) para aplicações de SLSM), os quais são sistemas cujos parametros são governados por uma cadeia de Markov subjacente $\Theta=\{\theta(t), t \geq 0\}$, com $\theta(t)$ tomando valores sobre $\mathcal{S}=\{1,2, \ldots, N\}$, os resultados e propriedades relativos à observabilidade e controlabilidade têm alcançado um paralelo completo com aqueles presentes na teoria de SLD.

Motivamos ao leitor o estudo de SLSM, não somente por suas aplicações mas também pelo fato que estes sistemas generalizam os já conhecidos SLD, apresentando resultados relevantes e propriedades análogas às daqueles sistemas. Entre outros, existem resultados importantes (vide Ji e Chizeck (1992); Morozan (1995); Costa e Fragoso (1995); Costa et al. (1999); Costa e Marques (2000) e Fragoso e Baczynski (2001)), referentes às equações algébricas de Riccati acompladas (EARA) correspondentes ao problema linear quadrático (LQ) e ao conceito S-estabilizabilidade, garantindo a existência de soluções das EARA. Citam-se para resultados que envolvem detetabilidade fraca (Wdetetabilidade) a Costa et al. (2005); do Val e Costa (2005); Costa e do Val (2002a,b); do Val e Costa (2002). Também há trabalhos tratando filtragem de SLSM nos quais se apresentam avanços recentes, vide de Souza et al. (2006); de Souza e Fragoso (2003); Fragoso e Rocha (2005); Fragoso et al. (2005), e interessantes avanços em Fragoso e Baczynski (2005); de Souza e Coutinho (2006); Fragoso e Costa (2005); Costa e Fragoso (2007); Dragan e Morozan (2008); Todorov e Fragoso (2008). Nota-se a relevancia que tem a pesquisa em SLSM e a diversidade de temas destacados acima.

\subsection{Hipóteses}

Vamos considerar inicialmente SLSM a tempo contínuo sendo definidos num espaço fundamental de probabilidade $\left(\Omega, \mathcal{F},\left\{\mathcal{F}_{t}\right\}, P\right)$ como

$$
\begin{aligned}
\Phi: \quad \dot{x}(t) & =A_{\theta(t)} x(t), \quad x\left(t_{0}\right)=x_{0}, \quad \theta\left(t_{0}\right) \sim \mu_{0} \\
y(t) & =C_{\theta(t)} x(t)
\end{aligned}
$$

com $t \geq 0, x \in \mathbb{R}^{n}$ e $y \in \mathbb{R}^{r}$. A cada instante de tempo $t$, temos $A_{\theta(t)}=A_{i}$ sempre que $\theta(t)=i$, sendo $A_{i}$ uma matriz de dimensão apropriada tomada de uma coleção conhecida de matrizes $A=\left(A_{1}, \ldots, A_{N}\right)$, e similarmente para $C$. A matriz de taxa de transição é denotada por $\Lambda=\left[\lambda_{i j}\right]$ (veja detalhes sobre cadeias de Markov em Bhattacharya e Waymire (1990)). Mencionamos 
as seguintes propriedades conhecidas para SLSM as quais têm uma contraparte direta para SLD:

(I) Subespaços não observados são invariantes, isto é, as trajetórias não observadas permanecem no espaço não observado quando as condições iniciais estão em tal espaço.

(II) Um sistema é observável se e somente se a coleção de matrizes de observabilidade $\mathcal{O}_{i}$ tem posto completo. ${ }^{1}$

(III) Observabilidade fraca (Costa e do Val (2002a)) é uma condição suficiente para que o controle LQ-ótimo seja estabilizante.

Veja Costa e do Val (2001, 2002a); Costa et al. (2005) para resultados adicionais sobre observabilidade fraca e a noção relacionada de detetabilidade fraca para SLSM e Meshkin e Khorasani (2009) para aplicações. A maioria dos resultados disponíveis (incluindo (I)-(III)) são baseados na propriedade seguinte: para cada $v \in \mathbb{R}^{n}, i \in \mathcal{S}$ e $k=0, \ldots, n^{2} N-1$

$$
\text { (P1): } \quad v^{\prime} O_{i}(k) v=0 \quad \Longleftrightarrow \quad O_{i}(k) v=0 .
$$

A propriedade (P1) é fácil de demonstrar no cenário a tempo discreto uma vez que $O_{i}(k)$ é uma matriz semidefinida positiva para cada $k$, vide Costa e do Val (2001). Porém, esse não é o caso a tempo contínuo. Assim, neste trabalho uma contribuição é uma prova para a propriedade (P1) para SLSM a tempo contínuo.

No que corresponde à controlabilidade, o sistema é perturbado na equação de estado por um ruído persistente e escrito na forma $d x(t)=A_{\theta(t)} x(t) d t+$ $B_{\theta(t)} d \zeta(t)$; para esse cenário adaptaremos as hipóteses dadas no caso da observabilidade e estenderemos a propriedade (P1) numa propriedade do tipo

$$
\text { (P2): } \quad v^{\prime} S_{i}(k) v=0 \quad \Longleftrightarrow \quad S_{i}(k) v=0
$$

sendo que as $S_{i}$ formam uma coleção de matrizes de controlabilidade.

\subsection{Contribuições}

- Demonstrar que $x(t), t \geq 0$, está no espaço nulo de $C_{j}$ quase certamente (q.c.) sempre que $j$ seja acessível de $\theta(0)$ e $x^{\prime} O_{\theta(0)}(k) x=0, k=$

\footnotetext{
${ }^{1}$ Vide o Capítulo 2 para a definição desta coleção de matrizes
} 
$0, \ldots, n^{2} N-1$, vide Corolário 2 .

- Explorar um resultado de invariância similar para $A_{\theta(t)}^{p} x(t), p \geq 0$, ou seja, demonstrar que as derivadas de $x(t)$ de qualquer ordem estão no espaço nulo de $C_{j}$, vide Lema 4 . Além disso, demonstrar que essa invariância ainda vale ao substituir $\theta(t) \operatorname{com} i$, assumindo $i$ acessível de $\theta(t)$, formalmente,

$$
C_{j} A_{i}^{p} v=0
$$

- Avaliar, para toda sequência de estados de Markov $i, i_{1}, \ldots, i_{m}$ tal que $\lambda_{i, i_{1}} \lambda_{i_{1}, i_{2}} \cdots \lambda_{i_{m-1}, i_{m}} \neq 0$, a validade de

$$
C_{i_{m}} A_{i_{m-1}}^{p_{1}} A_{i_{m-2}}^{p_{2}} \cdots A_{i}^{p_{m}} v=0
$$

sempre que $v^{\prime} O_{i}(k) v=0, k=0, \ldots, n^{2} N-1$.

- Usar termos como no lado esquerdo de (1.3) (envolvidos na definição de $\mathcal{O}_{i}$ ) para demonstrar que $\mathcal{O}_{i} v=0$ por inspeção direta, completando assim a prova da necessidade de (P1) (a suficiência é trivial).

- Introduzir uma coleção de matrizes de controlabilidade e a definição de sistema controlável a partir dessa coleção.

- Explorar o espaço não controlável e sua relação com um funcional de custo definido.

- Avaliar, para todas as sequências de estados de Markov $j, j_{1}, \ldots, j_{m}, i$ tal que

$\lambda_{j, j_{1}} \lambda_{j_{1}, j_{2}} \cdots \lambda_{j_{m}, i} \neq 0$, a validade de

$$
R_{j} A_{j}^{p_{m}} \cdots A_{j_{m-1}}^{p_{2}} A_{j_{m}}^{p_{1}} v=0
$$

sempre que $v^{\prime} S_{i}(k) v=0, k=0,1, \ldots, n^{2} N-1$ e com $R_{j}$ sendo matrizes relacionadas com a matriz de covariância do ruído .

- Demonstrar por inspeção direta a necessidade da propriedade (P2) usando termos como no lado esquerdo de (1.4). 


\subsection{Organização do Trabalho}

Este trabalho está organizado como segue. O Capítulo 2 fornece notações e alguns resultados preliminares. O Capítulo 3 apresenta alguns resultados de invariância quanto à matriz de observabilidade os quais são usados no resultado principal. O Capítulo 4 contem adaptações e extensões ao caso da matriz de controlabilidade. Finalmente, o Capítulo 5 apresenta algumas conclusões. 


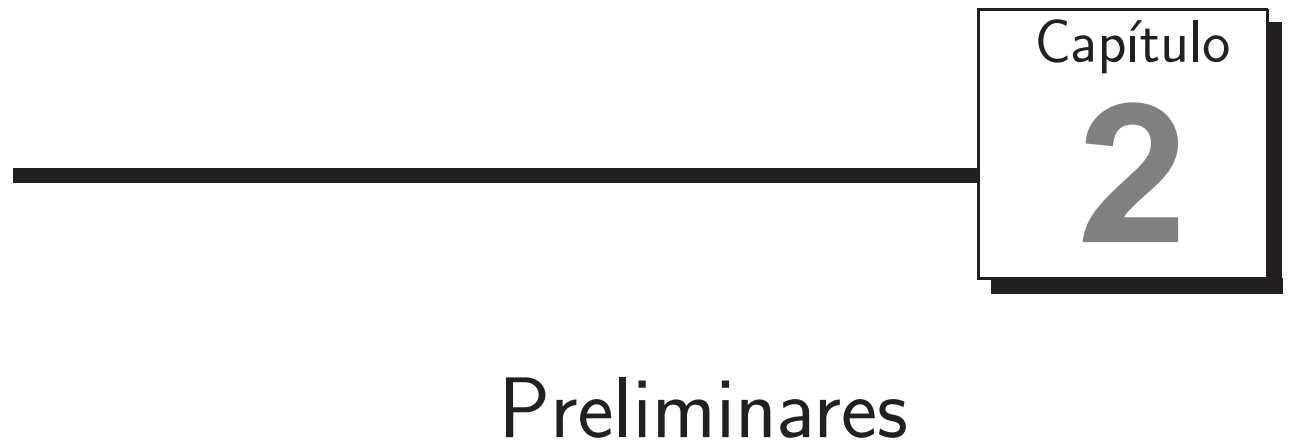

\subsection{Notações}

Nesta seção, apresentam-se notações e resultados para referência posterior. Seja $\mathcal{R}^{n, q}$ (respectivamente $\mathcal{R}^{n}$ ) o espaço linear formado por todas as matrizes de dimensão $n \times q$ (respectivamente, $n \times n)$ e $\mathcal{R}^{r 0}\left(\mathcal{R}^{r+}\right)$ o cone convexo fechado das matrizes simétricas semidefinidas positivas $\left\{U \in \mathcal{R}^{r}: U=U^{\prime} \geq 0\right\}$, (o cone aberto das matrizes simétricas definidas positivas $\left\{U \in \mathcal{R}^{r}: U=U^{\prime}>0\right\}$ ), onde $U^{\prime}$ denota a transposta de $U ; U \geq V(U>V)$ significa que $U-V \in R^{r 0}$ $\left(U-V \in R^{r+}\right)$. Para $U \in \mathcal{R}^{n, q}, \mathcal{N}(U)$ representa o espaço nulo de $U$. O operador $1_{\{.\}}$é a função indicadora (ou função característica) e $\operatorname{tr}\{$.$\} denota o$ traço. Seja $\mathcal{M}^{r, n}$ o espaço linear formado por um número $N$ de matrizes tal que $\mathcal{M}^{r, n}=\left\{U=\left(U_{1}, \ldots, U_{N}\right): U_{i} \in \mathcal{R}^{r, n}, i=1, \ldots, N\right\} ;$ também, $\mathcal{M}^{r} \equiv \mathcal{M}^{r, r}$. Denota-se por $\mathcal{M}^{r 0}\left(\mathcal{M}^{r+}\right)$ o conjunto $\mathcal{M}^{r}$ quando está formado por $U_{i} \in \mathcal{R}^{r 0}$ $\left(U_{i} \in \mathcal{R}^{r+}\right)$ para todo $i=1, \ldots, N . \mathcal{M}^{r, n}$ definido como antes junto com o produto escalar dado por

$$
\langle U, V\rangle=\sum_{j=1}^{n} \operatorname{tr}\left\{U_{j}^{\prime} V_{j}\right\}
$$

é um espaço de Hilbert (Costa e Fragoso (2005)). Além disto, define-se a norma $\|U\|=\langle U, I\rangle$ em $\mathcal{M}^{n 0}$, e, considerando o sistema $\Phi$ com $t_{0} \geq 0$, para 
$i=1, \ldots, N$ define-se:

$$
X_{i}(t)=E\left\{x(t) x(t)^{\prime} 1_{\{\theta(t)=i\}} \mid \mathcal{F}_{t_{0}}\right\}, \quad t \geq t_{0} .
$$

Considere os operadores $\mathcal{L}: \mathcal{M}^{n} \longrightarrow \mathcal{M}^{n}$ e $\mathcal{T}: \mathcal{M}^{n} \longrightarrow \mathcal{M}^{n}$ definidos como:

$$
\begin{aligned}
& \mathcal{L}_{i}(U)=A_{i}^{\prime} U_{i}+U_{i} A_{i}+\sum_{j=1}^{N} \lambda_{i j} U_{j}, \\
& \mathcal{T}_{i}(U)=A_{i} U_{i}+U_{i} A_{i}^{\prime}+\sum_{j=1}^{N} \lambda_{j i} U_{j}
\end{aligned}
$$

para $i=1, \ldots, N$. Denota-se $\mathcal{L}^{0}(U)=U$ e, para $k \geq 1, \mathcal{L}^{k}(U)=\mathcal{L}\left(\mathcal{L}^{k-1}(U)\right)$. Seja também $L(t), t \geq 0$, definido pela equação linear diferencial:

$$
\dot{L}_{i}(t)=\mathcal{L}_{i}(L(t))+C_{i}^{\prime} C_{i}, \quad L(0)=0, \quad t \geq 0
$$

para cada $i \in \mathbb{S}$. Considere agora o funcional

$$
W^{t_{0}, t}(x, \theta)=E\left\{\int_{t_{0}}^{t_{0}+t} x(\tau)^{\prime} C_{\theta(\tau)}^{\prime} C_{\theta(\tau)} x(\tau) d \tau \mid \mathcal{F}_{t_{0}}\right\}
$$

definido sempre que $x\left(t_{0}\right)=x$ e $\theta\left(t_{0}\right)=\theta$. Na continuação serão apresentadas outras notações adicionais e alguns resultados. Para $V \in \mathcal{R}^{n}$, as colunas de $V$ serão identificadas como $V=\left[v_{1} \vdots v_{2} \vdots \ldots \vdots v_{n}\right]$ e, para $U=\left(U_{1}, U_{2}, \ldots, U_{N}\right)$ introduzimos o já conhecido operador linear inversível $\hat{\varphi}: \mathcal{M}^{n} \longrightarrow \mathbb{R}^{n^{2} N}$,

$$
\hat{\varphi}(U)=\left[\begin{array}{c}
\varphi\left(U_{1}\right) \\
\varphi\left(U_{2}\right) \\
\vdots \\
\varphi\left(U_{N}\right)
\end{array}\right], \quad \operatorname{com} \quad \varphi(V)=\left[\begin{array}{c}
v_{1} \\
v_{2} \\
\vdots \\
v_{n}
\end{array}\right]
$$

Assim sendo, obtem-se que

$$
\hat{\varphi}(\mathcal{L}(U))=\mathcal{A} \hat{\varphi}(U)
$$


e $\mathcal{A}$ é definida como

$$
\mathcal{A}=\left[\begin{array}{cccc}
\hat{A}_{1}+\lambda_{11} I_{n^{2}} & \lambda_{11} I_{n^{2}} & \ldots & \lambda_{1 N} I_{n^{2}} \\
\lambda_{21} I_{n^{2}} & \hat{A}_{2}+\lambda_{22} I_{n^{2}} & \ldots & \lambda_{11} I_{n^{2}} \\
\vdots & \vdots & \ddots & \vdots \\
\lambda_{N 1} I_{n^{2}} & \lambda_{N 2} I_{n^{2}} & \ldots & \hat{A}_{N}+\lambda_{N N} I_{n^{2}}
\end{array}\right]
$$

com $\hat{A}_{i}=I_{n} \otimes A_{i}^{\prime}+A_{i}^{\prime} \otimes I_{n}$, sendo $\otimes$ o símbolo que representa o produto de Kronecker entre matrizes conforme Costa e do Val (2002a). Agora, ao aplicar o operador $\hat{\varphi}$ em (2.2) e utilizar (2.4), obtemos

$$
\begin{aligned}
\dot{\ell}(t) & =\hat{\varphi}\left[C^{\prime} C+\mathcal{L}(L(t))\right] \\
& =v+\mathcal{A} \hat{\varphi}(L(t))=v+\mathcal{A} \ell(t), \quad t \geq 0,
\end{aligned}
$$

sendo $\ell(t), q \in \mathbb{R}^{n^{2} N}$, definidos por

$$
\ell(t)=\hat{\varphi}(L(t)), \quad v=\hat{\varphi}\left(C^{\prime} C\right) .
$$

Inspecionando a relação anterior, notamos que

$$
\frac{d^{k} \ell}{d t^{k}}(0)=\mathcal{A}^{k} v
$$

\subsection{Definições e resultados}

Na continuação apresentaremos algumas definições e resultados iniciais que nos levaram ao resultado principal enunciado no Capítulo 3. Os seguintes resultados aparecem em Costa e do Val (2002a).

Proposição 1. As seguintes afirmações são válidas:

$$
W^{t_{0}, t}\left(x\left(t_{0}\right), \theta\left(t_{0}\right)\right)=\int_{0}^{t}\left\langle X(\tau), C^{\prime} C\right\rangle d \tau=\langle U, L(t)\rangle
$$

sempre que $U_{i}=x\left(t_{0}\right) x\left(t_{0}\right)^{\prime}$ para $\theta\left(t_{0}\right)=i$ e $U_{j}=0, j \neq i$.

A proposição prévia permite generalizar a definição do funcional $W$ como segue:

$$
W^{t_{0}, t}(U)=\int_{0}^{t}\left\langle X(\tau), C^{\prime} C\right\rangle d \tau=\langle U, L(t)\rangle, \quad \text { para } \quad U \in \mathcal{M}^{n}
$$


Também, introduzimos a seguinte representação para $\langle U, L(t)\rangle$ :

$$
\langle U, L(t)\rangle=\hat{\varphi}(U)^{\prime} \ell(t)
$$

Um resultado preliminar importante é establecido na seguinte proposição (veja Costa e do Val (2002a)). Pode ser interpretado como uma avaliação para a expansão máxima da trajetória $x(t)$ em torno de um estado dado $x_{0}$, e isto se segue dos fatos que a trajetória é contínua e tem derivadas limitadas $\left(A_{\theta(t)}\right.$ são tomadas da coleção finita $A$ conhecida).

Proposição 2. Para cada escalar $M>0$, existe $t_{M}>0$ tal que $\left\|x(t)-x_{0}\right\| \leq$ $M\left\|x_{0}\right\|$ (q.c.), $0 \leq t \leq t_{M}$.

Desenvolvemos o seguinte lema preliminar.

Lema 1. Se $W^{t+T}(x, i)=0$ então $W^{t, T}(x(t), \theta(t))=0$ (q.c.) para todo $t \geq 0$.

Demonstração. Vamos supor $W^{t, T}(x(t), \theta(t))>0$ com probabilidade positiva. Então temos que $E\left\{W^{T}(x(t), \theta(t)) \mid \mathcal{F}_{0}\right\}>0$, permitindo avaliar

$$
\begin{array}{rl}
W^{t+T}(x, i)=E & \left\{\int_{0}^{t} x(\tau)^{\prime} C_{i}^{\prime} C_{i} x(\tau) d \tau \mid \mathcal{F}_{0}\right\} \\
& +E\left\{\int_{t}^{t+T} x(\tau)^{\prime} C_{i}^{\prime} C_{i} x(\tau) d \tau \mid \mathcal{F}_{0}\right\} \\
\geq E & E\left\{W^{t, T}(x(t), \theta(t)) \mid \mathcal{F}_{0}\right\}>0 .
\end{array}
$$

Definição 1 (Matriz de Observabilidade). A coleções de matrizes $\mathcal{O}_{i} \in \mathcal{M}^{n\left(n^{2} N\right), n}$ definidas para cada $i \in \mathcal{S}$ como

$$
\mathcal{O}_{i}=\left[\begin{array}{llll}
O_{i}^{\prime}(0) & O_{i}^{\prime}(1) \quad \ldots & O_{i}^{\prime}\left(n^{2} N-1\right)
\end{array}\right]^{\prime}
$$

é denominada conjunto de matrizes de observabilidade do sistema $\Phi$, sendo $O(k) \in \mathcal{M}^{n}$ definida para cada $i \in \mathcal{S}$ como

$$
O_{i}(k)=\frac{d^{k+1} L_{i}}{d t^{k+1}}(0)
$$

com $L(t) \in \mathcal{M}^{n}, t \geq 0$, definida pelas equações lineares dadas em (2.2) com $O_{i}(0)=C_{i}^{\prime} C_{i}$ para cada $i \in \mathcal{S}$. Se $\mathcal{O}_{i}$ é de posto completo para cada $i$ então o sistema é observável. 
$O_{i}(k)$ pode não ser positiva já que, de outra forma (se for positiva para $t>0) L_{i}(t)$ deveria tender para infinito e portanto, $W^{\infty}(x, i)=\infty$.

Note que $O(k)$ pode ser definido equivalentemente, de forma recursiva, usando o operador $\mathcal{L}$ :

$$
O_{i}(k)=\mathcal{L}_{i}(O(k-1)), \quad k>0
$$

Exemplo 2. Considere o SLSM $\Phi$ contínuo no tempo com

$$
\begin{gathered}
A_{1}=\left[\begin{array}{ccc}
2 & \delta & 0 \\
0.01 & 1 & 0 \\
0.9 & \delta & 0.1
\end{array}\right], \quad C_{1}=\left[\begin{array}{lll}
1 & 0 & 0
\end{array}\right], \\
A_{2}=\left[\begin{array}{ccc}
0.5 & 0 & 0 \\
0 & 0.99 & 0 \\
1 & 0 & 1
\end{array}\right], \quad C_{2}=\left[\begin{array}{lll}
0 & 0 & 0
\end{array}\right], \\
A_{3}=\left[\begin{array}{ccc}
1 & 0 & 0 \\
0.1 & 0.25 & 0 \\
0 & \delta & 1
\end{array}\right], \quad C_{3}=C_{1}, \quad C_{4}=0.3 C_{1} \\
A_{4}=0.1\left[\begin{array}{ccc}
3 & 0.4 \delta & 0.1 \\
0 & 1 & 0 \\
2 & 0 & 1
\end{array}\right], \quad \Lambda=\left[\begin{array}{cccc}
-3 & 0.9 & 2 & 0.1 \\
2 & -5 & 2 & 1 \\
0.5 & 0.5 & -1 & 0 \\
0 & 0 & 0 & 0
\end{array}\right] .
\end{gathered}
$$

Note que $\mathcal{S}=\{1,2,3,4\}, n=3$ e $N=3$. Se $\delta \neq 0$, ao calcular a matriz de observabilidade $\mathcal{O}$ obtemos que, para cada $i=1,2,3,4$, as matrizes $\mathcal{O}_{i}$ são de posto $=3$ sendo assim o sistema observável. No entanto, ao fazer $\delta=0 e$ calcular a matriz de observabilidade vemos que para cada $i$ as matrizes $\mathcal{O}_{i}$ tem posto $=2$ e portanto o sistema é não-observável. Observe que $\delta$ nulo reflete na não-observabilidade do sistema.

Lema 2. ${ }^{1}$ Sejam $x \in \mathbb{R}^{n}, i \in \mathcal{S}, X \in \mathcal{M}^{n}$ definida como $X_{i}=x x^{\prime}$ e $X_{j}=0$, $\forall j \neq i$ e $w \in \mathbb{R}^{n^{2} N}$ definida como $w=\hat{\varphi}(X)$. As seguintes afirmações são equivalentes:

(i) $w^{\prime} \frac{d^{k+1} \ell}{d t^{k+1}}(0)=0$, para $k=0, \ldots, n^{2} N-1$.

(ii) $x \in \mathcal{N}\left(L_{i}(t)\right)$ ou, equivalentemente, $w^{\prime} \ell(t)=0$ para todo $t \geq 0$.

\footnotetext{
${ }^{1}$ Resultado extraído de Costa e do Val (2002a).
} 


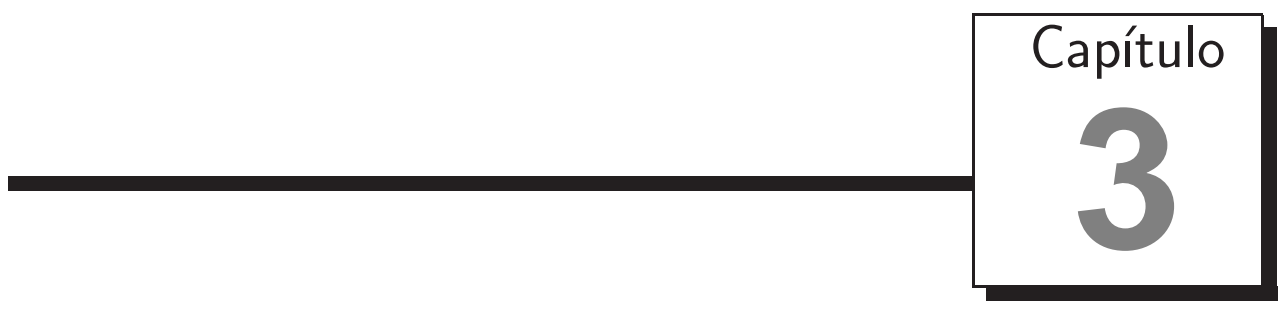

Matriz de Observabilidade e propriedades de invariância

\subsection{Considerações e resultados iniciais}

Neste capítulo desenvolvemos propriedades de invariância associadas à observabilidade, já comentadas na Seção 1.3. Pretendemos, então, empregar esses resultados na demonstração da equivalência das afirmações (i) e (ii) do Lema 2 e o fato de $x$ pertencer a $\mathcal{N}\left(\mathcal{O}_{i}\right)$, como é proposto em Costa e do Val (2002a). Porém, para este fim, necessitaremos de alguns resultados preliminares. O primeiro é uma adaptação de (Costa e do Val, 2002a, Lemma 8).

Corolário 1. $x^{\prime} O_{i}(k) x=0$ para todo $k=0, \ldots, n^{2} N-1$, se e somente se $W^{t}(x, i)=0, t \geq 0$.

Demonstração. Seja $X \in \mathcal{M}^{n}$ definido como $X_{i}=x x^{\prime}$ e $X_{j}=0, \forall j \neq i$ e $w \in \mathbb{R}^{n^{2} N}$ como $w=\hat{\varphi}(X)$. As seguintes implicações são obtidas de uma maneira simples para cada $k=0, \ldots, n^{2} N-1$,

$$
\begin{aligned}
x^{\prime} O_{i}(k) x=0 \Leftrightarrow x^{\prime} \frac{d^{k+1} L}{d t^{k+1}}(0) x=0 & \Leftrightarrow\left\langle X, \frac{d^{k+1} L}{d t^{k+1}}(0)\right\rangle=0 \\
& \Leftrightarrow w^{\prime} \frac{d^{k+1} \ell}{d t^{k+1}}(0)=0 .
\end{aligned}
$$

De (3.1) e o Lema 2 temos que $x^{\prime} O_{i}(k) x=0, k=0, \ldots, n^{2} N-1$, é equivalente 
a $w^{\prime} \ell(t)=0, t \geq 0, \operatorname{logo}(2.7)$ e $(2.8)$ completam a prova.

Na continuação exploramos um resultado de invariância para a trajetória $x(t)$ e os espaços nulos associados a $C$.

Lema 3. Se existe $T$ tal que $x(T) \notin \mathcal{N}\left(C_{j}\right)$ com probabilidade positiva, $1 \leq$ $j \leq N$, então existe $l \geq 0$ tal que $W^{l+s}(x(T), \theta(T))>0$ (q.c.), s $\geq 0$, sempre que $j$ seja acessivel de $\theta(T)$.

Demonstração. Nesta prova definiremos um intervalo $\left[T, t_{\epsilon}\right]$ para permitir $\theta(T)$ alcançar $j$ com probabilidade positiva e um intervalo de tempo $\left[t_{\epsilon}, t_{\epsilon}+s\right]$ para integração do termo $x(t)^{\prime} C_{j}^{\prime} C_{j} x(t)$ e assim, obtemos avaliações para a mínima distancia entre a trajetória de $x(t)$ e o espaço nulo de $C_{j}$ durante esses intervalos de tempo, vide Figura 3.1. Se $x(T) \notin \mathcal{N}\left(C_{j}\right)$, temos que $\|x-x(T)\| \geq m$, $\forall x \in \mathcal{N}\left(C_{j}\right)$, onde $0<m=d\left(x(T), \mathcal{N}\left(C_{j}\right)\right)$. Agora, para $\epsilon=\frac{m}{2\|x(T)\|}$, existe $\delta_{\epsilon}>0$ tal que

$$
x(t) \in B\left(x(T), \frac{m}{2}\right) \quad \text { (q.c.), } \quad \operatorname{com} T \leq t \leq t_{\epsilon}=T+\delta_{\epsilon},
$$

(vide Proposição 2). Seja $\tilde{x}\left(t_{\epsilon}\right)$ e $\hat{x}\left(t_{\epsilon}\right)$ as projeções ortogonais de $x\left(t_{\epsilon}\right)$ em $\mathcal{N}\left(C_{j}\right)$ e $\mathcal{N}\left(C_{j}\right)^{\perp}$ respectivamente. Assim, $x\left(t_{\epsilon}\right)=\tilde{x}\left(t_{\epsilon}\right)+\hat{x}\left(t_{\epsilon}\right)$ e, portanto, existe $\mu>0$ tal que:

$$
\begin{aligned}
x\left(t_{\epsilon}\right)^{\prime} C_{j}^{\prime} C_{j} x\left(t_{\epsilon}\right) & =\hat{x}\left(t_{\epsilon}\right)^{\prime} C_{j}^{\prime} C_{j} \hat{x}\left(t_{\epsilon}\right) \geq \mu\left\|\hat{x}\left(t_{\epsilon}\right)\right\|^{2} \\
& \geq \mu\left(\left\|\tilde{x}\left(t_{\epsilon}\right)-x(T)\right\|-\left\|x\left(t_{\epsilon}\right)-x(T)\right\|\right)^{2} \\
& \geq \mu \frac{m^{2}}{4} \quad \text { (q.c.). }
\end{aligned}
$$

Numa maneira similar obtemos para $s>0$,

$$
x(\tau)^{\prime} C_{j}^{\prime} C_{j} x(\tau) \geq \mu \frac{m^{2}}{16} \text { (a.s.), } \quad t_{\epsilon} \leq \tau \leq t_{\epsilon}+s,
$$

o que produz

$$
\int_{t_{\epsilon}}^{s+t_{\epsilon}} x(\tau)^{\prime} C_{j}^{\prime} C_{j} x(\tau) d \tau \geq \mu \frac{m^{2}}{16} s \quad \text { (q.c.) }
$$

isto é,

$$
\left.W^{s}\left(x\left(t_{\epsilon}\right), j\right) \geq \mu \frac{m^{2}}{16} s \quad \text { (q.c. }\right) \text {. }
$$




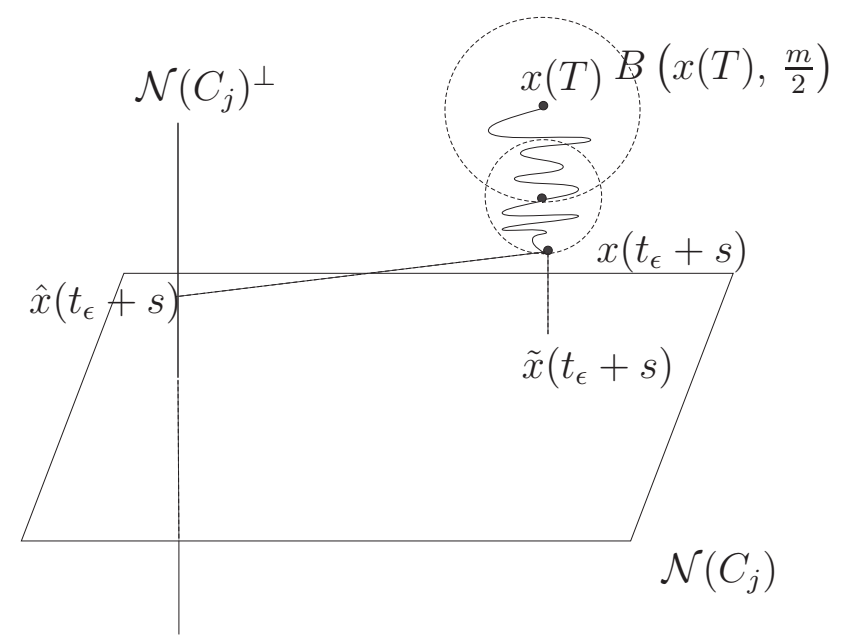

Figura 3.1: Cenário da prova do Lema 3 ilustrando os intervalos de tempo considerados e a análise de pior caso para a trajetória de $x(t)$ com respeito à distância a $\mathcal{N}\left(C_{j}\right)$.

Agora, avaliamos

$$
\begin{aligned}
E\left\{W^{s}\left(x\left(t_{\epsilon}\right), \theta\left(t_{\epsilon}\right)\right) \mid \mathcal{F}_{T}\right\} & \geq E\left\{W^{s}\left(x\left(t_{\epsilon}\right), j\right) 1_{\left\{\theta\left(t_{\epsilon}\right)=j\right\}} \mid \mathcal{F}_{T}\right\} \\
& \geq \mu \frac{m^{2}}{16} s E\left\{1_{\left\{\theta\left(t_{\epsilon}\right)=j\right\}} \mid \mathcal{F}_{T}\right\}>0
\end{aligned}
$$

onde a última desigualdade segue do fato que $j$ é acessível de $\theta(T)$. Finalmente,

$$
\begin{aligned}
\int_{T}^{s+t_{\epsilon}} x(\tau)^{\prime} C_{j}^{\prime} C_{j} x(\tau) d \tau & =\int_{T}^{t_{\epsilon}} x(\tau)^{\prime} C_{j}^{\prime} C_{j} x(\tau) d \tau \\
& \quad+\int_{t_{\epsilon}}^{s+t_{\epsilon}} x(\tau)^{\prime} C_{j}^{\prime} C_{j} x(\tau) d \tau \\
& \geq \int_{t_{\epsilon}}^{s+t_{\epsilon}} x(\tau)^{\prime} C_{j}^{\prime} C_{j} x(\tau) d \tau
\end{aligned}
$$

levando em

$$
\begin{aligned}
W^{s+t_{\epsilon}}(x(T), \theta(T)) & =E\left\{W^{s+t_{\epsilon}}(x(T), \theta(T)) \mid \mathcal{F}_{T}\right\} \\
& \geq E\left\{W^{s}\left(x\left(t_{\epsilon}\right), \theta\left(t_{\epsilon}\right)\right) \mid \mathcal{F}_{T}\right\}>0 \text { (q.c.) } .
\end{aligned}
$$

Corolário 2. Se $W^{t}(x, i)=0$ para todo $t \geq 0$ então $C_{i_{m}} x(t)=0$ (q.c.), $\forall t \geq 0$ sempre que $i_{m}$ seja acessível de $i$. 
Demonstração. Uma vez que $W^{t}(x, i)=0$ para qualquer $t \geq 0$, em particular temos para algum $s \geq 0$ fixo e $r \geq 0$ que $W^{t+s+r}(x, i)=0, \forall t \geq 0$, e usando o Lema 1 obtemos que

$$
W^{s+r}(x(t), \theta(t))=0 \quad \text { (q.c.), } \quad \forall t \geq 0 \text {. }
$$

Logo, Lema 3 leva a

$$
x(t) \in \mathcal{N}\left(C_{i_{m}}\right) \quad \text { (q.c.) } \quad \text { para todo } t \geq 0,
$$

ou, equivalentemente,

$$
C_{i_{m}} x(t)=0 \quad \text { (q.c.), } \quad \forall t \geq 0 \text {. }
$$

O seguinte fato básico deverá ser empregado no resultado principal.

Lema 4. Para $Q \in \mathcal{R}^{m, n}$, temos $Q A_{\theta(t)}^{n} x(t)=0$ (q.c.) $t \geq 0, n>0$, sempre que $Q x(t)=0$ (q.c.) $t \geq 0$.

Demonstração. Para qualquer $v \in \mathcal{N}(Q)^{\perp}$ temos $v^{\prime} x(t)=0$ (q.c.), $\forall t \geq 0$. Agora, para todo $t \geq 0$ e $n>0$ avaliamos:

$$
\frac{d^{n}\left(v^{\prime} x(t)\right)}{d t^{n}}=v^{\prime}\left(\frac{d^{n} x(t)}{d t^{n}}\right)=0 \quad \text { (q.c.) }
$$

ou, equivalentemente, $d^{n} x(t) / d t^{n} \in \mathcal{N}(Q)$ (q.c.), $t \geq 0$, implicando

$$
Q A_{\theta(t)}^{n} x(t)=0 \quad \text { (q.c.), } \quad \forall t \geq 0
$$

Os resultados precedentes nos permitem demonstrar que, dado $x^{\prime} \mathcal{O}_{i} x=0$, então $Q A_{\theta(t)}^{n} x(t)=0$ (q.c.), $t \geq 0$. No entanto, gostariamos de generalizar essa avaliação a qualquer estado de Markov $i_{m}$ acessível de $\theta(t)$.

Lema 5. Assuma que a sequência de estados de Markov $\theta(t), i_{1}, i_{2}, \ldots, i_{m-1}, i_{m}$ é tal que $\lambda_{\theta(t), i_{1}} \lambda_{i_{1}, i_{2}} \cdots \lambda_{i_{m-1}, i_{m}} \neq 0$. Se $Q A_{\theta(t)}^{p} x(t)=0$ (q.c.), $t \geq 0$, então $Q A_{i_{m}}^{p} x(t)=0$ (q.c.), $t \geq 0, p \geq 0$. 
Demonstração. Assumiremos o contrário, isto é, que existe $T$, tal que $Q A_{i_{m}}^{p} x(T) \neq 0$ com probabilidade positiva $\alpha$, ou seja, $P(x(T) \notin \mathcal{N})=\alpha>0$, onde definimos $\mathcal{N}=\mathcal{N}\left(Q A_{i_{m}}^{p}\right)$. Então, $\forall \epsilon>0, \exists t_{\epsilon}>0$ (possivelmente dependente de $x(T))$ satisfazendo:

$$
Q A_{i_{m}}^{p} x(l) \neq 0, \quad \text { (q.c.) } T<l \leq T+t_{\epsilon} .
$$

Agora, assumimos que $T_{1}$ seja o tempo da primeira visita ao estado $i_{m}$ começando de $T$, isto é, $T_{1}=\inf _{t \geq 0}\left\{t \geq T: \theta(t)=i_{m}\right\}-T$, então temos que $P\left(T_{1}<t_{\epsilon}\right)>0$. Assim,

$$
\begin{aligned}
P\left(Q A_{\theta(l)}^{p} x(l) \neq 0\right)= & P\left(Q A_{\theta(l)}^{p} x(l) \neq 0 \mid x(T) \in \mathcal{N}\right)(1-\alpha) \\
& +P\left(Q A_{\theta(l)}^{p} x(l) \neq 0 \mid x(T) \notin \mathcal{N}\right) \alpha \\
\geq & P\left(Q A_{\theta(l)}^{p} x(l) \neq 0 \mid x(T) \notin \mathcal{N}\right) \alpha
\end{aligned}
$$

e, $\operatorname{com} l=T_{1}$ obtemos,

$$
\begin{aligned}
& P\left(Q A_{\theta\left(T_{1}\right)}^{p} x\left(T_{1}\right) \neq 0\right) \geq P\left(Q A_{\theta\left(T_{1}\right)}^{p} x\left(T_{1}\right) \neq 0 \mid x(T) \notin \mathcal{N}\right) \alpha \\
& \quad \geq P\left(Q A_{\theta\left(T_{1}\right)}^{p} x\left(T_{1}\right) \neq 0 \mid x(T) \notin \mathcal{N}, T_{1}<t_{\epsilon}\right) \alpha P\left(T_{1}<t_{\epsilon}\right) \\
& \quad=\alpha P\left(T_{1}<t_{\epsilon}\right)>0,
\end{aligned}
$$

o qual contradiz a hipótese que $Q A_{\theta(t)}^{p} x(t)=0$ (q.c.), $t \geq 0$.

O Lema 4 pode ser empregado para obter a avaliação chave para a necessidade de (P1), como segue.

Corolário 3. Para cada $x \in \mathbb{R}^{n}$ e cada sequência de estados de Markov $i, i_{1}, \ldots, i_{m}$ tal que $x^{\prime} O_{i}(k) x=0, k=0, \ldots, n^{2} N-1$, e $\lambda_{i, i_{1}} \lambda_{i_{1}, i_{2}} \cdots \lambda_{i_{m-1}, i_{m}} \neq$ 0 , temos

$$
C_{i_{m}} A_{i_{m-1}}^{p_{1}} A_{i_{m-2}}^{p_{2}} \cdots A_{i}^{p_{m}} x=0
$$

para cada inteiro $p_{\ell} \geq 0, \ell=1, \ldots, m$.

Demonstração. Se para cada $k=0, \ldots, n^{2} N-1$ nós temos $x^{\prime} O_{i}(k) x=0$ então, segundo os Corolários 1 e 2 obtemos

$$
C_{i_{m}} x(t)=0 \quad \text { (q.c.), } \quad t \geq 0
$$

e também (vide Lema 4) 


$$
C_{i_{m}} A_{\theta(t)}^{p_{1}} x(t)=0 \quad \text { (q.c.), } \quad \forall t \geq 0 .
$$

Assuma que $\theta(t)=i$ (desde que $\theta(0)=i$ temos que $\theta(t)=i$ com probabilidade positiva). Por hipótese, $\theta(t)$ alcança $i_{m-1}$ e, assim, aplicando o Lema 5 obtemos,

$$
C_{i_{m}} A_{i_{m-1}}^{p_{1}} x(t)=0 \quad \text { (q.c.), } \quad \forall t \geq 0 .
$$

Se fazemos $Q=C_{i_{m}} A_{i_{m-1}}^{p_{1}}$, então procedendo similarmente como antes (substituindo $C_{i_{m}}$ por $Q$ em (3.3)) obtemos

$$
Q A_{\theta(t)}^{p_{2}} x(t)=0 \quad \text { (q.c.) } \quad \forall t \geq 0
$$

e, lembrando que assumimos $\theta(t)=i$, temos novamente por hipótese que $\theta(t)$ alcança $i_{m-2}$, levando em

$$
C_{i_{m}} A_{i_{m-1}}^{p_{1}} A_{i_{m-2}}^{p_{2}} x(t)=0 \quad \text { (q.c.) } \quad \forall t \geq 0 .
$$

Procedendo recursivamente,

$$
C_{i_{m}} A_{i_{m-1}}^{p_{1}} A_{i_{m-2}}^{p_{2}} \cdots A_{i}^{p_{m}} x(t)=0 \quad \text { (q.c.) } \quad \forall t \geq 0
$$

e, em particular para $t=0$, podemos ver que (3.2) se cumpre.

O seguinte exemplo ilustra o resultado no Corolário 3.

Exemplo 3. Considere o SLSM $\Phi$ contínuo no tempo com

$$
\begin{array}{ll}
A_{1}=\left[\begin{array}{ccc}
2 & 0 & 0 \\
0.01 & 1 & 0 \\
0.9 & 1 & 0.1
\end{array}\right], \quad C_{1}=\left[\begin{array}{lll}
1 & 0 & 0 \\
0 & 0 & 0 \\
0 & 0 & 0
\end{array}\right], \\
A_{2}=\left[\begin{array}{ccc}
0.5 & 0 & 0 \\
0 & 0.99 & 0 \\
1 & 1 & 1
\end{array}\right], \quad C_{2}=\left[\begin{array}{lll}
0 & 1 & 0 \\
0 & 0 & 0 \\
0 & 0 & 0
\end{array}\right],
\end{array}
$$




$$
A_{3}=\left[\begin{array}{ccc}
1 & 0 & 0 \\
0.1 & 0.25 & 0 \\
0 & 0 & 1
\end{array}\right], \quad C_{3}=0, \quad \Lambda=\left[\begin{array}{ccc}
-3 & 1 & 2 \\
2 & -5 & 3 \\
0.5 & 0.5 & -1
\end{array}\right]
$$

Note que $\mathcal{S}=\{1,2,3\}, n=3, N=3$. Consideramos a condição inicial $(x(0), \theta(0))$ com $x(0)=\left[\begin{array}{lll}0 & 0 & 1\end{array}\right]^{\prime}$ e $\theta(0)=1$ (compativel com a distribuição inicial $\left.\mu_{0}=\left[\begin{array}{lll}1 & 0 & 0\end{array}\right]\right)$. Considere a sequência $1,3,1,2,3,2$, para a qual $\lambda_{1,3} \lambda_{3,1} \lambda_{1,2} \lambda_{2,3} \lambda_{3,2} \neq 0$. Verificamos que $x^{\prime} O_{\theta(0)}(k) x=0, k=0, \ldots, 26$, satisfazendo assim as hipóteses do Corolário 3, e

$$
C_{2} A_{3}^{p_{1}} A_{2}^{p_{2}} A_{1}^{p_{3}} A_{3}^{p_{4}} A_{1}^{p_{5}} x(0)=0
$$

para cada $p_{\ell}=0, \ldots, 20, \ell=1, \ldots, 5$. Isto confirma (3.2).

Exemplo 4. Consideremos o sistema do Exemplo 2 no Capítulo 2 e assuma a condição inicial $(x(0), \theta(0))$ como no exemplo anterior. Como vimos o sistema é não-observável se $\delta=0$ nas matrizes $A_{1}, A_{3}$ e $A_{4}$. É gerada a sequência de estados de Markov 1,3,1,3,1,2,4. Note que ao chegar a cadeia no estado $i=4$ ela não volta mais para os estados 1,2 ou 3 pois $\lambda_{4, j}=0$ para $j=1,2,3,4$. Assim, segundo o Corolário 3, já que o sistema é não-observável e a sequência de estados $1,3,1,3,1,2,4$ é tal que $\lambda_{1,3} \lambda_{3,1} \lambda_{1,3} \lambda_{3,1} \lambda_{1,2} \lambda_{2,4} \neq 0$ podemos então verificar que

$$
C_{4} A_{2}^{p_{1}} A_{1}^{p_{2}} A_{3}^{p_{3}} A_{1}^{p_{4}} A_{3}^{p_{5}} A_{1}^{p_{4}} x(0)=0 .
$$

Se supomos agora uma cadeia de estados dada por 1,3,1,3,1,2,4,3 onde claramente temos que $\lambda_{1,3} \lambda_{3,1} \lambda_{1,3} \lambda_{3,1} \lambda_{1,2} \lambda_{2,4} \lambda_{4,3}=0$ então observamos que

$$
C_{3} A_{4}^{p_{1}} A_{2}^{p_{2}} A_{1}^{p_{3}} A_{3}^{p_{4}} A_{1}^{p_{5}} A_{3}^{p_{6}} A_{1}^{p_{7}} x(0)=\left[\begin{array}{lll}
1.31789 & 0 & 0.00001
\end{array}\right]\left[\begin{array}{l}
0 \\
0 \\
1
\end{array}\right] \neq 0
$$

\subsection{Suficiência de (P1)}

Rescrevemos abaixo a propriedade (P1) para facilitar a leitura,

$$
(\mathrm{P} 1): \quad v^{\prime} O_{i}(k) v=0 \quad \Longleftrightarrow \quad O_{i}(k) v=0 .
$$

A necessidade de (P1) segue por avaliação direta de $\mathcal{O}_{i} x$ com a ajuda do Corolário 3. De fato, de (2.1), (2.2) e (2.10) pode-se verificar que $O_{i}(k)$ é uma 
soma envolvendo termos da forma

$$
\pi A_{i}^{\prime q_{k}} A_{j_{1}}^{\prime q_{k-1}} \cdots A_{j_{k-1}}^{\prime q 1} C_{i_{k}}^{\prime} C_{i_{k}} A_{i_{k-1}}^{p_{1}} A_{i_{k-2}}^{p_{2}} \cdots A_{i}^{p_{k}} x
$$

onde $i, i_{1}, \ldots, i_{k}$ e $i, j_{1}, \ldots, j_{k-1}, i_{k}$ são sequências de estados de Markov, $p_{1}+\cdots+p_{k} \leq k, q_{1}+\cdots+q_{k} \leq k$, e $\pi$ aparece no produtorio das taxas relacionadas às transições $i, i_{1}, \ldots, i_{k}$ and $i, j_{1}, \ldots, j_{k-1}, i_{k}$. Pode-se verificar também que $\pi$ é diferente de zero se e somente se $\lambda_{i, i_{1}} \lambda_{i_{1}, i_{2}} \cdots \lambda_{i_{k-1}, i_{k}} \neq 0$ e $\lambda_{i, j_{1}} \lambda_{j_{1}, j_{2}} \cdots \lambda_{j_{k-1}, i_{k}} \neq 0$. Então, assumindo $x^{\prime} O_{i}(k) x=0$ para cada $k=$ $0, \ldots, n^{2} N-1$, e $\pi \neq 0$, Corolário 3 implica que $C_{i_{k}} A_{i_{k-1}}^{p_{1}} A_{i_{k-2}}^{p_{2}} \cdots A_{i}^{p_{k}} x=0$, numa maneira tal que o termo (3.4) é zero. Como uma ilustração, assumimos $x^{\prime} O_{i}(k) x=0, k=0, \ldots, n^{2} N-1$, e realizamos as avaliações para $O_{i}(k)$, $k=0,1,2$. Temos que

$$
O_{i}(0)=C_{i}^{\prime} C_{i}
$$

e segue imediatamente do Corolário 3 que $C_{i} x=0$, daqui que $O_{i}(0) x=$ $C_{i}^{\prime} C_{i} x=0$. De (2.1), (2.2) e (2.10),

$$
O_{i}(1)=A_{i}^{\prime} C_{i}^{\prime} C_{i}+C_{i}^{\prime} C_{i} A_{i}+\sum_{j=1}^{N} \lambda_{i j} C_{j}^{\prime} C_{j}+C_{i}^{\prime} C_{i}
$$

e pós-multiplicando cada termo de $O_{i}(1)$ por $x$ e usando novamente o Corolário 3 obtemos $O_{i}(1) x=0$. Agora,

$$
\begin{aligned}
O_{i}(2)= & A_{i}^{\prime 2} C_{i}^{\prime} C_{i}+2 A_{i}^{\prime} C_{i}^{\prime} C_{i} A_{i}+A_{i}^{\prime} \sum_{j=1}^{N} \lambda_{i j} C_{j}^{\prime} C_{j}+A_{i}^{\prime} C_{i}^{\prime} C_{i} \\
& +C_{i}^{\prime} C_{i} A_{i}^{2}+\left(\sum_{j=1}^{N} \lambda_{i j} C_{j}^{\prime} C_{j}\right) A_{i}+C_{i}^{\prime} C_{i} A_{i}+C_{i}^{\prime} C_{i} \\
& +\sum_{j=1}^{N} \lambda_{i j}\left(A_{j}^{\prime} C_{j}^{\prime} C_{j}+C_{j}^{\prime} C_{j} A_{j}+\sum_{s=1}^{N} \lambda_{j s} C_{s}^{\prime} C_{s}+C_{j}^{\prime} C_{j}\right)
\end{aligned}
$$

Similarmente acima, Corolário 3 produz $O_{i}(2) x=0$. Continuando com esse processo demonstramos o seguinte.

Teorema 1. Para cada $k=0, \ldots, n^{2} N-1$ temos que $x^{\prime} O_{i}(k) x=0$ se e somente se $O_{i}(k) x=0$.

Neste exemplo avaliamos a matriz $\mathcal{O}_{i}$ e verificamos que $\mathcal{O}_{i} x=0$ dado que $x^{\prime} O_{i}(k) x=0$, em concordancia com o Teorema 1 . 
Exemplo 5. Considere o sistema $\Phi$ como no Exemplo 3, com $C_{2}=0 . A$ condição inicial permanece sem ser alterada, $\theta(0)=1$ e $x(0)=\left[\begin{array}{lll}0 & 0 & 1\end{array}\right]^{\prime}$. Realizamos o cálculo de $O_{i}(k), k=0, \ldots, 26$, e $\mathcal{O}_{i}$ via (2.1) e (2.2). O espaço nulo de $\mathcal{O}$ coincide com o espaço gerado pelos vetores $v_{1}=\left[\begin{array}{lll}0 & 1 & 0\end{array}\right]^{\prime} e$ $v_{2}=\left[\begin{array}{ccc}0 & 0 & 1\end{array}\right]^{\prime} ;$ os elementos de $O_{i}(k)$ são todos nulos exceto o elemento $(1,1)$, numa maneira tal que $x^{\prime} O_{i}(k) x=0$ se e somente se $x$ pertence ao espaço nulo gerado pelos vetores $v_{1}, v_{2}$, confirmando o Teorema 1 . Note que $x(0) \in \mathcal{N}\left(\mathcal{O}_{i}\right)$, de uma forma tal que $W^{t}(x(0), 1)=0$ (vide Corolário 1), e as hipóteses do Corolário 2 são satisfeitas para $i_{m}=1,2,3$; a Figura 3.2 confirma que $x(t), t \geq 0$, está no espaço nulo de $C_{1}$ (obviamente, o mesmo acontece para $C_{2}=C_{3}=0$ ). 

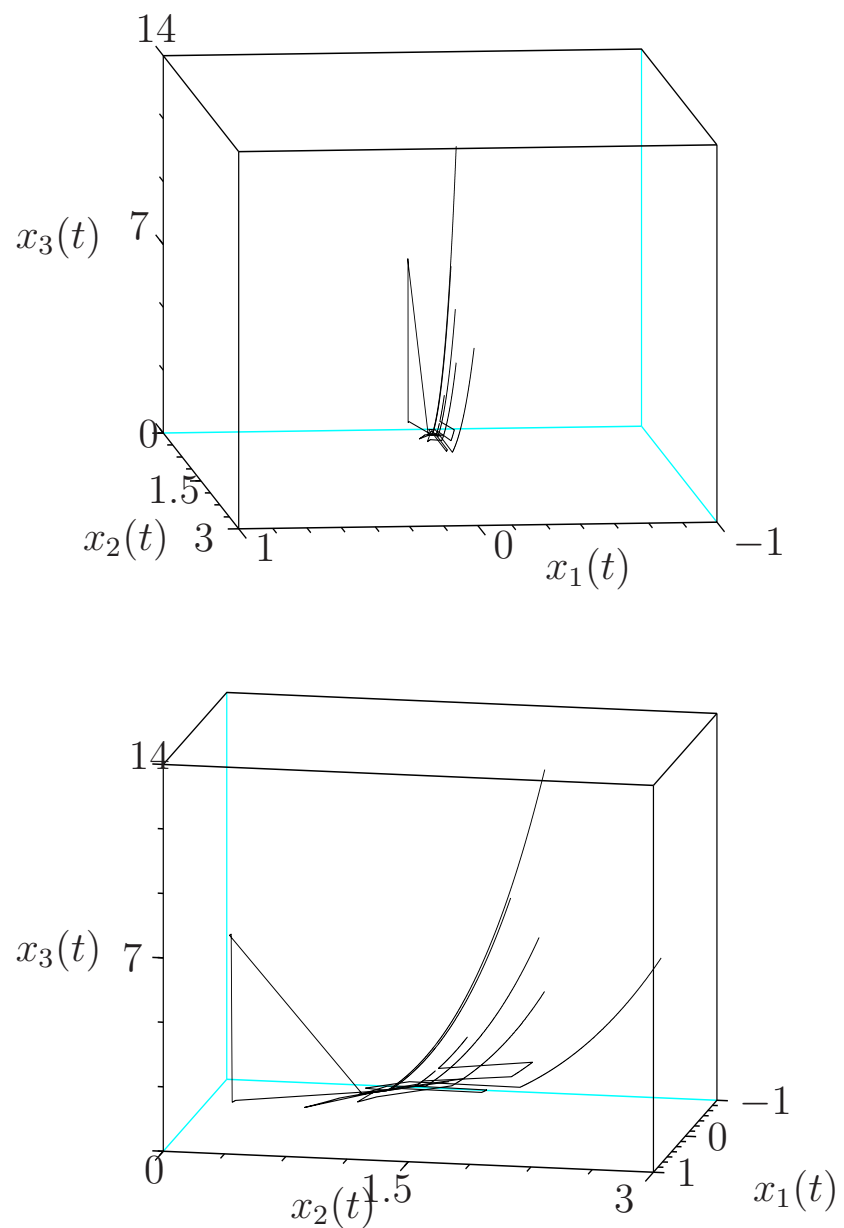

Figura 3.2: Duas diferentes perspectivas de 10 trajetórias de $x(t), 0 \leq t \leq S$, onde $S$ corresponde ao instante do quinto salto, para o sistema no Exemplo 5. Cada trajetória corresponde a uma realização de $\theta$. 
Capítulo

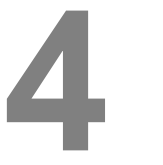

\section{Extensões e/ou adaptações à matriz de Controlabilidade}

\subsection{Adaptações e definições iniciais}

Consideremos o seguinte sistema:

$$
\begin{aligned}
\Psi: \quad \dot{x}(t) & =A_{\theta(t)} x(t)+B_{\theta(t)} \zeta(t), \quad x\left(t_{0}\right)=x_{0}, \quad \theta\left(t_{0}\right) \sim \pi \\
y(t) & =C_{\theta(t)} x(t)
\end{aligned}
$$

sendo $x(t) \in \mathbb{R}^{n}, \zeta(t) \in \mathbb{R}^{q}, y(t) \in \mathbb{R}^{m}$. Assumimos neste capítulo que a distribuição inicial é invariante, isto é, $\dot{\pi}=0$ (por exemplo no caso de cadeia de Markov ergódica tomamos $\left.\pi_{0}=\pi_{\infty}\right)$.

Vamos considerar o operador $\mathcal{T}: \mathcal{M}^{n} \longrightarrow \mathcal{M}^{n}$ em (2.1) dado como:

$$
\mathcal{I}_{i}(U)=A_{i} U_{i}+U_{i} A_{i}^{\prime}+\sum_{j=1}^{N} \lambda_{j i} U_{j}
$$

para $i=1, \ldots, N$. Recordando que $X(t) \in \mathcal{M}^{n}$ é tal que $X_{i}(t)=E\left\{x(t) x(t)^{\prime} 1_{\{\theta(t)=i\}} \mid \mathcal{F}_{0}\right\}, i=1, \ldots, N$ e $t \geq 0$, então temos a seguinte equação diferencial (vide Costa e Fragoso (2005):

$$
\dot{X}(t)=\mathcal{T}(X(t))+R, \quad X(0)=0
$$


onde $R \in \mathcal{M}^{n 0}, R_{i}=B_{i} \hat{R} B_{i}^{\prime} \pi_{i}$, e $\hat{R}$ é a matriz de covariância do processo $\{\zeta(t): t \geq 0\})$. Vamos definir a sequência de coleções de matrizes $S_{i}(k)$ para cada $i=1, \ldots, N$ por:

$$
S_{i}(k)=\frac{d^{k+1} X_{i}}{d t^{k+1}}(0), \quad S_{i}(0)=R_{i}
$$

Introduzimos também a matriz

$$
\mathcal{C}_{i}=\left[\begin{array}{llll}
S_{i}(0) & S_{i}(1) & \ldots & S_{i}\left(n^{2} N-1\right)
\end{array}\right]
$$

que chamamos matriz de controlabilidade.

Observação 1. Note que a matriz definida acima corresponde a uma formulação dual à observabilidade.

O objetivo é demonstrar a Propriedade (P2). De (4.2), nós vemos que ao aplicar o operador $\hat{\varphi}$ obtemos:

$$
\hat{\varphi}(\dot{X}(t))=\mathcal{A}^{\prime} \hat{\varphi}(X(t))+\hat{\varphi}(R)
$$

e, se definimos $z(t)=\hat{\varphi}(X(t))$ e $r=\hat{\varphi}(R)$ temos que

$$
z(t)=\int_{0}^{t} e^{\mathcal{A}^{\prime} t} r d \tau
$$

\subsection{Resultados auxiliares}

Para auxiliar no desenvolvimento das demonstrações, consideremos agora o sistema sem ruído persistente

$$
\begin{aligned}
\dot{\epsilon}(t) & =A_{\theta(t)} \epsilon(t) \quad \epsilon\left(t_{0}\right)=\epsilon_{0}, \quad \theta\left(t_{0}\right) \sim \mu_{0} \\
y(t) & =C_{\theta(t)} \epsilon(t)
\end{aligned}
$$

sendo $\epsilon_{0}$ tal que $\mathcal{E}_{i}(0)=E\left\{\epsilon_{0} \epsilon_{0}^{\prime} 1_{\{\theta(t)=i\}}\right\}=Q_{i}, i=1, \ldots, N$. Com isso, temos a seguinte equação diferencial (agora homogênea, veja Costa e Fragoso (2005)):

$$
\dot{\mathcal{E}}(t)=\mathcal{T}(\mathcal{E}(t)), \quad \mathcal{E}(0)=Q,
$$


cuja solução é dada como (aplicando novamente o operador $\varphi$ em (4.7) e fazendo as substituições $w(t)=\hat{\varphi}(\mathcal{E}(t)), q=\hat{\varphi}(Q))$ :

$$
w(t)=e^{\mathcal{A}^{\prime} t} q
$$

Também consideramos o funcional definido como

$$
W^{t}=\int_{0}^{t}\left\langle\mathcal{E}(t), C^{\prime} C\right\rangle d t, \quad t \geq 0
$$

Para enfatizar a dependência de $W$ em $Q$ e $C$, denotamos $W^{t}(Q, C)$. Agora, suponha que $Q=\sum_{j=1}^{N} \tilde{Q}_{j}$, sendo $\tilde{Q}_{j}=\left(0, \ldots, 0, R_{j}, 0, \ldots, 0\right)$ e cada $R_{j}$ é componente da matriz $R$ que aparece em (4.2) e é da forma $R_{j}=\sum_{k=1}^{n} r_{j k} r_{j k}^{\prime}$. Portanto, $\tilde{Q}_{j}=\sum_{k=1}^{n} \tilde{R}_{j k}$ com $\tilde{R}_{j k}=\left(0, \ldots, 0, r_{j k} r_{j k}^{\prime}, 0, \ldots, 0\right)$, notando que o termo não nulo encontra-se na $j$-ésima posição; finalmente, $Q=\sum_{j=1}^{N} \sum_{k=1}^{n} \tilde{R}_{j k}$. Com isto, (4.8) pode ser escrita também como

$$
w(t)=e^{\mathcal{A}^{\prime} t} \sum_{j=1}^{N} \sum_{k=1}^{n} \hat{\varphi}\left(\tilde{R}_{j k}\right) .
$$

Também, para cada $j=1, \ldots, N$ e $k=1, \ldots, n$, introduzimos as variáveis $\tilde{X}_{j k} \in \mathcal{M}^{n 0}$ tais que:

$$
\dot{\tilde{X}}_{j k}(t)=\mathcal{T}\left(\tilde{X}_{j k}(t)\right), \quad \tilde{X}_{j k}(0)=\tilde{R}_{j k}
$$

De fato podemos definir sistemas $\Phi_{j k}$ tais que $\dot{\delta}(t)=A_{\theta(t)} \delta(t), \quad \delta\left(t_{0}\right)=$ $\delta_{0}, \quad \theta\left(t_{0}\right) \sim \mu_{0} ; \quad y(t)=C_{\theta(t)} \delta(t)$, sendo $\delta_{0}$ tal que $\Delta_{j k}(0)=E\left\{\delta_{0} \delta_{0}^{\prime} 1_{\{\theta(t)=i\}}\right\}=$ $\tilde{R}_{j k}, j=1, \ldots, N$. A solução de (4.11) (usando mais uma vez o operador $\varphi$ e fazendo $\left.z_{j k}(t)=\hat{\varphi}\left(\tilde{X}_{j k}(t)\right)\right)$ vem dada por $z_{j k}(t)=e^{\mathcal{A}^{\prime} t} \hat{\varphi}\left(\tilde{R}_{j k}\right)$. Assim, ao 
calcular $W^{t}(Q, C)$ temos que,

$$
\begin{aligned}
W^{t}(Q, C) & =W^{t}\left(\sum_{j=1}^{N} \tilde{R}_{j}, C\right)=\sum_{j=1}^{N} W^{t}\left(\tilde{R}_{j}, C\right) \\
& =\sum_{j=1}^{N} W^{t}\left(\sum_{k=1}^{n} \tilde{R}_{j k}, C\right)=\sum_{j=1}^{N} \sum_{k=1}^{n} W^{t}\left(\tilde{R}_{j k}, C\right) \\
& =\sum_{j=1}^{N} \sum_{k=1}^{n} \int_{0}^{t}\left\langle\tilde{X}_{j k}(\tau), C^{\prime} C\right\rangle d \tau \\
& =\sum_{j=1}^{N} \sum_{k=1}^{n} \int_{0}^{t} z_{j k}(\tau)^{\prime} \hat{\varphi}\left(C^{\prime} C\right) d \tau \\
& =\int_{0}^{t} \sum_{j=1}^{N} \sum_{k=1}^{n} z_{j k}(\tau)^{\prime} \hat{\varphi}\left(C^{\prime} C\right) d \tau=\int_{0}^{t} w(\tau)^{\prime} \hat{\varphi}\left(C^{\prime} C\right) d \tau\left(\int^{t} \int_{0}^{\mathcal{A}^{\prime} t} q\right. \\
& =\hat{\varphi}\left(C^{\prime} C\right)^{\prime} \int_{0}^{t} w\left(\tau=\hat{\varphi}\left(C^{\prime} C\right)^{\prime} \int_{0}\right.
\end{aligned}
$$

Assim, quando $Q=R$, de (4.5) e (4.17) temos que

$$
W^{t}(R, C)=\hat{\varphi}\left(C^{\prime} C\right)^{\prime} z(t)
$$

de forma que calcular o funcional de custo no sistema $\Phi$ é equivalente ao produto interno da solução $z(t)$ associada ao sistema $\Psi$ com a direção $v=$ $\hat{\varphi}\left(C^{\prime} C\right)$.

Observação 2. Observe que $\Phi$ em (4.6) é o mesmo sistema $\Phi$ do Capítulo 2 quando $\epsilon_{0}=x_{0}$. É simples verificar que se fizermos $\epsilon_{0}=x_{0}=r_{j k}$ e $\mu_{0}$ for tal que $\mu_{0}(i)=1, i \neq j$ e $\mu_{0}(j)=0$, temos que $W^{t}\left(\tilde{R}_{j k}, C\right)=W^{t}\left(r_{j k}, j\right)$ sendo este último custo definido em (2.3).

\subsection{Resultados de Controlabilidade.}

Lema 6. Considere o sistema $\Psi$ e assuma $\hat{\varphi}\left(C^{\prime} C\right)^{\prime} z(t)=0$ para todo $t \geq 0$. Para cada $j \in \mathbb{S}$ para os quais existem sequências de Markov $j, j_{1}, \ldots, j_{m}, i$ $\operatorname{com} \lambda_{j, j_{1}} \lambda_{j_{1}, j_{2}} \cdots \lambda_{j_{m}, i} \neq 0$ (j alcança $i$ ) temos que

$$
R_{j} A_{j}^{\prime p_{m}} \cdots A_{j_{m-1}}^{p_{2}} A_{j_{m}}^{p_{1}} C_{i}^{\prime}=0
$$

Demonstração. $\hat{\varphi}\left(C^{\prime} C\right)^{\prime} z(t)=0$ é equivalente a $W^{t}(\hat{R}, C)=0$ no sistema 
$\Phi$, que, por sua vez, implica em $W^{t}\left(r_{j k}, j\right)=0$, para $j=1, \ldots, N, k=$ $1, \ldots, n$. Então se $j$ alcança $i$, pelo Corolário 3 no problema de observabilidade, $W^{t}\left(r_{j k}, j\right)=0$ implica em

$$
C_{i} A_{j_{m}}^{p_{1}} A_{j_{m-1}}^{p_{2}} \cdots A_{j}^{p_{m}} r_{j k}=0
$$

o qual é equivalente a

$$
C_{i} A_{j_{m}}^{p_{1}} A_{j_{m}}^{p_{2}} \cdots A_{j}^{p_{m}} \sum_{k=1}^{n} r_{j k} r_{j k}^{\prime}=0
$$

Por definição de $R_{j}$ e transposição finalmente temos

$$
R_{j} A_{j}^{\prime p_{m}} \cdots A_{j_{m-1}}^{p_{2}} A_{j_{m}}^{\prime p_{1}} C_{i}^{\prime}=0
$$

como queria-se provar.

Adiante, para $v \in \mathbb{R}^{n}, i \in \mathbb{S}$, vamos definir $C \in \mathcal{M}^{n, m}$ tal que $C_{i}=v^{\prime}$ e $C_{j}=0$ para $j \neq i$. Alguns resultados análogos àqueles em Costa e do Val (2002a) são apresentados na continuação.

Proposição 3. As seguintes afirmações são equivalentes:

(i) $v^{\prime} X_{i}^{\prime}(s) v=0$ para algum $s \geq 0$.

(ii) $\hat{\varphi}\left(C^{\prime} C\right)^{\prime} \frac{d^{m} z}{d t^{m}}(0)=0$ para $m=1,2, \ldots$

(iii) $\hat{\varphi}\left(C^{\prime} C\right)^{\prime} \mathcal{A}^{\prime m-1} r=0$ para $m=1,2, \ldots$

(iv) $\hat{\varphi}\left(C^{\prime} C\right)^{\prime} z(t)=0$ e $v \in \mathcal{N}\left(X_{i}(t)\right)$, para todo $t \geq 0$.

Demonstração. $(i) \Rightarrow(i i) . X(t) \leq X(s)$, para $0 \leq t \leq s$, então:

$$
z(t)^{\prime} \hat{\varphi}\left(C^{\prime} C\right)=\left\langle X(t), C^{\prime} C\right\rangle \leq\left\langle X(s), C^{\prime} C\right\rangle \leq v^{\prime} X_{i}(s) v=0
$$

Logo,

$$
z(t)^{\prime} \hat{\varphi}\left(C^{\prime} C\right)=0, \quad \text { para } \quad 0 \leq t \leq s
$$

e, portanto,

$$
\hat{\varphi}\left(C^{\prime} C\right)^{\prime} \frac{d^{m} z}{d t^{m}}(0)=0 \quad \text { para } \quad m=1,2, \ldots
$$

$(i i) \Rightarrow(i i i) . \hat{\varphi}\left(C^{\prime} C\right)^{\prime} \frac{d^{m} z}{d t^{m}}(0)=\mathcal{A}^{\prime m-1} r, m=1,2, \ldots$, leva ao resultado.

$($ iii $) \Rightarrow($ iv $)$. Temos que $X(t)=S^{\prime} S$ para alguma matriz $S$ não singular. 
Então:

$$
\begin{aligned}
X_{i}(t) v=0 \quad \forall t \geq 0 & \Leftrightarrow \quad S^{\prime} S v=0 \Leftrightarrow v^{\prime} S^{\prime} S v=0 \Leftrightarrow v^{\prime} X_{i}(t) v=0 \quad \forall t \geq 0 \\
& \Leftrightarrow\left\langle X(t), C^{\prime} C\right\rangle=0 \quad \forall t \geq 0, \Leftrightarrow \hat{\varphi}\left(C^{\prime} C\right)^{\prime} z(t)=0,
\end{aligned}
$$

para todo $t \geq 0$.

Mostremos então que $\hat{\varphi}\left(C^{\prime} C\right)^{\prime} z(t)=0 \quad \forall t \geq 0$ :

$$
z(t)=\int_{0}^{t} e^{\mathcal{A}^{\prime} \tau} r d \tau=\int_{0}^{t}\left(\sum_{k=0}^{\infty} \frac{\left(\mathcal{A}^{\prime} \tau\right)^{k}}{k !}\right) r d \tau=\int_{0}^{t}\left(\sum_{k=0}^{\infty} \frac{\tau^{k}}{k !} \mathcal{A}^{\prime k}\right) r d \tau,
$$

$\log \mathrm{O}$

$$
\hat{\varphi}\left(C^{\prime} C\right)^{\prime} z(t)=\sum_{k=0}^{\infty}\left[\int_{0}^{t}\left(\frac{\tau^{k}}{k !} d \tau\right) \hat{\varphi}\left(C^{\prime} C\right)^{\prime} \mathcal{A}^{\prime k} r\right]=0,
$$

e portanto temos que $v \in \mathcal{N}\left(X_{i}(t)\right) \quad \forall t \geq 0$.

$(i v) \Rightarrow(i)$. Trivial.

Lema 7. $v^{\prime} S_{i}(k) v=0, k=0,1, \ldots$, se e somente se $W^{t}(R, C)=0$ para todo $t \geq 0$.

Demonstração. Para $k=0,1, \ldots$ temos:

$$
v^{\prime} S_{i}(k) v=0 \quad \Leftrightarrow \quad v^{\prime} \frac{d^{k+1} X_{i}}{d t^{k+1}}(0) v=0 \quad \Leftrightarrow \quad \hat{\varphi}\left(C^{\prime} C\right)^{\prime} \frac{d^{k+1} z}{d t^{k+1}}(0)=0
$$

A última expressão acima, pela proposição anterior é equivalente a $\hat{\varphi}\left(C^{\prime} C\right)^{\prime} z(t)=0, \forall t \geq 0$, que pela sua vez é equivalente a $W^{t}(R, C)=0, \forall t \geq$ 0 (vide (4.17)).

Corolário 4. $v^{\prime} S_{i}(k) v=0, k=0,1, \ldots$ implica em $R_{j} A_{j}^{\prime p_{m}} \cdots A_{j_{m-1}}^{\prime p_{2}} A_{j_{m}}^{\prime p_{1}} v=0$ para todo $j$ que alcança $i$.

Demonstração. Se $v^{\prime} S_{i}(k) v=0, k=0,1, \ldots$, o Lema 7 leva em $W^{t}(R, C)=0$ e o Lema 6 implica em $R_{j} A_{j}^{\prime p_{m}} \cdots A_{j_{m-1}}^{p_{2}} A_{j_{m}}^{\prime p_{1}} C_{i}^{\prime}=0$ para $i$ accesível de $j$. Usando a construção de $C^{\prime} C$ acima temos finalmente que $R_{j} A_{j}^{\prime p_{m}} \cdots A_{j_{m-1}}^{\prime p_{2}} A_{j_{m}}^{\prime p_{1}} v=0$.

Podemos observar, de forma análoga que no caso da observabilidade, que $S_{i}(k)$ é também uma soma envolvendo termos da forma

$$
\sigma A_{i_{m}}^{q_{1}} A_{i_{m-1}}^{q_{2}} \cdots A_{j}^{q_{m}} R_{j} A_{j}^{p_{m}} \cdots A_{j_{m-1}}^{p_{2}} A_{j_{m}}^{p_{1}} v
$$


sendo $j, j_{1}, \ldots, j_{m}, i$ e $j, i_{1}, \ldots, i_{m}, i$ sequências de estados de Markov. Então, assumindo $v^{\prime} S_{i}(k) v=0$ para cada $k=0, \ldots, n^{2} N-1$, e $\sigma \neq 0$, Corolário 4 produz que $R_{j} A_{j}^{\prime p_{m}} \cdots A_{j_{m-1}}^{p_{2}} A_{j_{m}}^{p_{1}} v=0$, de tal forma que (4.19) é zero.

Teorema 2. Considere o sistema $\Psi$ e seja $i \in \mathbb{S}$. Para cada $k=0,1, \ldots, n^{2} N-$ 1 temos que $S_{i}(k) v=0$ se e somente se $v^{\prime} S_{i}(k) v=0$. 


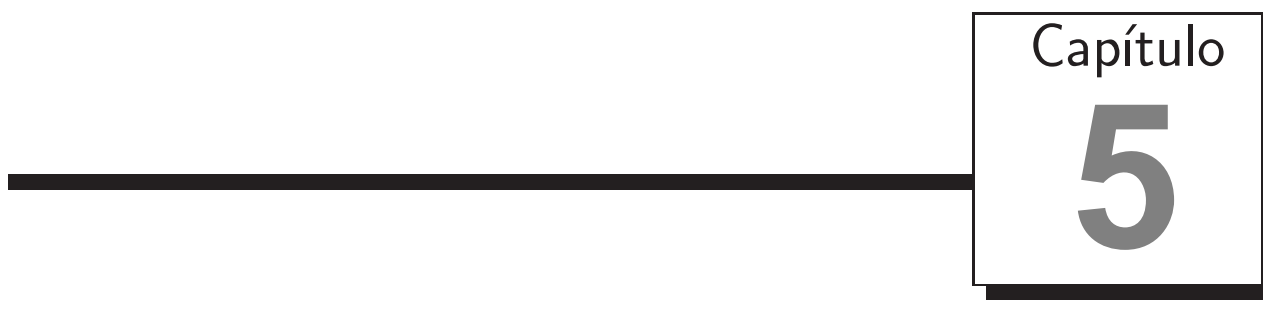

\section{Conclusões}

Neste trabalho foram estabelecidos alguns resultados de invariância para a trajetória $x(t)$, incluindo a avaliação apresentada no Corolário 3 na forma

$$
C_{i_{m}} A_{i_{m-1}}^{p_{1}} A_{i_{m-2}}^{p_{2}} \cdots A_{i}^{p_{m}} x=0
$$

sempre que $x$ é tal que $x^{\prime} O_{i}(k) x=0, k=0, \ldots, n^{2} N-1$, e sequência de estados de Markov $i, i_{1}, \ldots, i_{m}$ satisfaz $\lambda_{i, i_{1}} \lambda_{i_{1}, i_{2}} \cdots \lambda_{i_{m-1}, i_{m}} \neq 0$, com a interpretação de que a aplicação de quaisquer potências das matrizes $A_{i_{k}}$ (associadas com derivadas de quaisquer ordens de $x$ ) não são capazes de retirar $x$ do espaço nulo de $C_{i}$.

Essa avaliação é empregada então para demonstrar a equivalência (P1) (vide Capítulo 3), a qual é essencial para as noções de obsevabilidade e detetabilidade fraca para SLSM contínuos no tempo e diversas propriedades, resultados e aplicações (veja Costa e do Val (2002a); Meshkin e Khorasani (2009)) que nela se baseiam.

Também estendemos os resultados de observabilidade ao cenário da controlabilidade, fazendo inicialmente algumas adaptações de definições, proposições, lemas, etc. Com isso, e com alguns resultados de observabilidade já estabelecidos, conseguimos demonstrar uma propriedade similar à propriedade (P1) envolvendo a matriz de controlabilidade. 


\section{Referências Bibliográficas}

Astolfi, A.; Praly, L. Global complete observability and output-to-state stability imply the existence of a globally convergent observer. Mathematics of Control, Signals and Systems, v. 18, p. 32-65, 2006.

Bhattacharya, R. B.; Waymire, E. C. Stochastic processes with aplications. Wiley-Interscience, 1990.

Bittanti, S.; Colaneri, P.; Guardabassi, G. N-controllability and observability of linear periodic systems. SIAM Journal on Control and Optimization, v. 22, p. 889-893, 1984.

Costa, E. F.; Do VAL, J. B. R. On the detectability and observability of discrete-time Markov jump linear systems. System and Control Letters, v. 44, p. $135-145,2001$.

Costa, E. F.; Do VAL, J. B. R. On the detectability and observability of continuous-time Markov jump linear systems. SIAM Journal on Control and Optimization, v. 41, n. 4, p. 1295-1314, 2002a.

Costa, E. F.; Do VAL, J. B. R. Weak detectability and the linear-quadratic control problem of discrete-time Markov jump linear systems. International Journal of Control, Special Issue on Switched and Polytopic Linear Systems, v. 75 , n. 16/17, p. 1282-1292, 2002b.

Costa, E. F.; do Val, J. B. R.; Fragoso, M. D. A new approach to detectability of discrete-time infinite Markov jump linear systems. SIAM Journal on Control and Optimization, v. 43, p. 2132-2156, 2005.

Costa, E. F.; Manfrim, A. L. P.; Do Val, J. B. R. Weak controllability and weak stabilizability concepts for linear systems with Markov jump 
parameters. In: Proc. ACC'06 American Control Conference, Minneapolis,USA, 2006.

Costa, O. L. V.; do Val, J. B. R.; Geromel, J. C. Continuoustime state-feedback $H_{2}$-control of markovian jump linear systems via convex analysis. Automatica, v. 35, p. 259-268, 1999.

Costa, O. L. V.; Fragoso, M. Discrete-time LQ-optimal control problems for infinite Markov jump parameter systems. IEEE Transactions on Automatic Control, v. AC-40, p. 2076-2088, 1995.

Costa, O. L. V.; Fragoso, M. D. A unified approach for stochastic and mean square stability of continuous-time linear systems with markovian jumping parameters and additive disturbances. SIAM journal on control and optimization, ISSN 0363-0129, v. 4, p. 1165-1191, 2005.

Costa, O. L. V.; Fragoso, M. D. A separation principle for the h-2control of continuous-time infinite Markov jump linear systems with partial observations. Journal of Mathematical Analysis and Applications, v. 331, n. 1, p. 97-120, 2007.

Costa, O. L. V.; Marques, R. Robust $H_{2}$-control for discrete-time markovian jump linear systems. International Journal of Control, v. 73, n. 1, p. $11-21,2000$.

Davis, M. H. A.; Vinter, R. B. Stochastic modelling and control. Chapman and Hall, 1984.

DE Souza, C. E.; Coutinho, D. F. Robust stability of a class of uncertain Markov jump nonlinear systems. IEEE Transactions on Automatic Control, v. 51, n. 11, p. 1825-1831, 2006.

DE Souza, C. E.; Fragoso, M. D. H-infinity filtering for discrete-time linear systems with markovian jumping parameters. International Journal of Robust and Nonlinear Control, v. 13, n. 14, p. 1299-1316, 2003.

de Souza, C. E.; Trofino, A.; Barbosa, K. A. Mode-independent $\mathrm{H}$-infinity filters for markovian jump linear systems. IEEE Transactions on Automatic Control, v. 51, n. 11, p. 1837-1841, 2006. 
do Val, J.; Costa, E. F. Numerical solution for the linear-quadratic control problem of Markov jump linear systems and a weak detectability concept. Journal of Optimization Theory and Applications, v. 114, n. 1, p. 69-96, 2002.

DO VAL, J. B. R.; BASAR, T. Receding horizon control of jump linear systems and a macroeconomic policy problem. Journal of Economic Dynamics and Control, v. 23, p. 1099-1131, 1999.

Do VAL, J. B. R.; Costa, E. F. Stabilizability and positiveness of solutions of the jump linear quadratic problem and the coupled algebraic Riccati equation. IEEE Transactions on Automatic Control, v. 50, n. 5, p. 691-695, 2005 .

Dragan, V.; Morozan, T. Exponential stability in mean square for a general class of discrete-time linear stochastic systems. Journal of Stochastic Analysis and Applications, v. 26, n. 3, p. 495-525, 2008.

Fragoso, M.; Baczynski, J. Optimal control for continuous time LQ problems with infinite Markov jump parameters. SIAM Journal on Control and Optimization, v. 40, n. 1, p. 270- 297, 2001.

Fragoso, M. D.; Baczynski, J. On an infinite dimensional perturbed Riccati differential equation arising in stochastic control. Linear Algebra and its Applications, v. 406, p. 165-176, 2005.

Fragoso, M. D.; Costa, O. L. V. A unified approach for stochastic and mean square stability of continuous-time linear systems with markovian jumping parameters and additive disturbances. SIAM Journal on Control and Optimization, v. 44, n. 4, p. 1165-1191, 2005.

Fragoso, M. D.; Costa, O. L. V.; Baczynski, J.; Rocha, N. C. S. Optimal linear mean square filter for continuous-time jump linear systems. IEEE Transactions on Automatic Control, v. 50, n. 9, p. 1364-1369, 2005.

Fragoso, M. D.; Rocha, N. C. S. Stationary filter for continuous-time markovian jump linear systems. SIAM Journal on Control and Optimization, v. 44, n. 3, p. 801-815, 2005.

Gray, W. S.; Mesko., J. P. Observability functions for linear and nonlinear systems. Systems and Control Letters, v. 38, p. 99-113, 1999. 
Ji, Y.; Chizeck, H. J. Controllability, observability and discrete-time markovian jump linear quadratic control. International Journal of Control, v. 48, n. 2, p. 481-498, 1988.

Ji, Y.; Chizeck, H. J. Controlability, stabilizability and continuous time Markovian jump linear quadratic control. IEEE Transactions on Automatic Control, v. 35, n. 7, p. 777-788, 1990.

Ji, Y.; Chizeck, H. J. Jump linear quadratic gaussian conrol in continuous time. IEEE Transactions on Automatic Control, v. 37, n. 12, p. 1884-1892, 1992.

Luenberger, D. G. Dynamic systems theory, models and applications. John Wiley \& Sons, 1979.

Meshinin, N.; Khorasani, K. Fault detection and isolation of discrete-time markovian jump linear systems with application to a network of multi-agent systems having imperfect communication channels. Automatica, v. 45, p. 2032-2040, 2009.

Morozan, T. Stability and control for linear systems with jump Markov perturbations. Stochastic Analysis and Applications, v. 13, n. 1, p. 91-110, 1995.

O. L. V. Costa, M. D. F.; Marques, R. P. Discrete-time markov jump linear systems. Springer, 2000.

Petersen, I. R. Notions of observability for uncertain linear systems with structured uncertainty. SIAM J. Control Optim., v. 41, n. 2, p. 345-361, 2002 .

SARIDIS, G. N. Intelligent robotic control. IEEE Transactions on Automatic Control, v. 28, p. 547-557, 1983.

Siqueira, A. A. G.; Terra, M. H. Nonlinear and markovian $\mathrm{H}_{\infty}$ controls of underactuated manipulators. IEEE Transactions on Control System Technology, v. 12, 2004.

Todorov, M. G.; Fragoso, M. D. Output feedback h-infinity control of continuous-time infinite markovian jump linear systems via lmi methods. SIAM Journal on Control and Optimization, v. 47, p. 950-974, 2008. 